\title{
Performance, flexibility and usability enhancement of the fiber optic vibration sensor
}

\author{
Vít Novotný ${ }^{1 *}$, Karel Slavíček ${ }^{2}$, Petr Sysel ${ }^{3}$, Aleš Prokeš ${ }^{4}$, and Pavel Hanák ${ }^{5}$
}

1 Faculty of Business and Management, Brno University of Technology, novotnyv@fbm.vutbr.cz.

${ }^{2}$ Faculty of Electrical Engineering and Communication, Brno University of Technology, slavicekkarel@feec.vutbr.cz.

${ }^{3}$ Faculty of Electrical Engineering and Communication, Brno University of Technology, sysel@feec.vutbr.cz.

${ }^{4}$ Faculty of Electrical Engineering and Communication, Brno University of Technology, prokes@feec.vutbr.cz.

${ }^{5}$ Faculty of Electrical Engineering and Communication, Brno University of Technology, hanakp@feec.vutbr.cz.

Correspondence should be addressed to Vít Novotný; novotnyv@fbm.vutbr.cz

\begin{abstract}
Distributed fiber optic vibration sensors based on $\Phi$-OTDR using Rayleigh backscattering of highly coherent light source pulses are very popular type in the area of sensors due to many attractive features, mainly due to its distributed manner of sensing, i.e. they can operate as thousands of local sensors simultaneously. The paper summarizes and describes several important modifications of the original sensor scheme to enhance its key performance parameters and to extend its application potential. A multi-fiber sensor form was proposed. Single- and two-fiber sensors were designed, constructed and tested both in the laboratory and in a real environment. Variants of two-fiber sensor utilizing optical switches for the sensor flexibility enhancement are proposed and their applications are described.
\end{abstract}

\section{Introduction}

Optical fibers are well known as brilliant electromagnetic waveguides that dominate internet communication technologies and are capable to transport data with the capacity up to terabits per second and per a fiber, [1]. The area of sensors where optical fibers are used as a sensing element is also widely and rapidly developing field of industry, [2]. There is a number of fiber -based sensors groups, they can be specified according to different points of view, e.g. into extrinsic and intrinsic sensor classes, point or distributed sensors, etc. The fiber-based sensors are used in many areas of industry, mainly for health structure monitoring, ([3], [4], [5], [6]) but its potential is also in a security area to detect, locate and optionally classify intruders. 
A distributed fiber optic vibration sensor based on $\Phi$-OTDR (phase-sensitive optical time domain reflectometry) is the most widespread sensor for sensing the occurrence of (generally multiple) mechanical vibrations with the ability to detect, locate, or, if needed to classify the activity of sources of mechanical vibration. This type of sensor has a wide range of applications in both the civilian and security sectors, and its research has been very intensive within more than twenty last years ([7] - [30]).

\section{Technology of Distributed Fiber Optic Vibration Sensing}

The principle of $\Phi$-OTDR sensors consists (see Figure 1) in the emission of narrow interrogating optical pulses down to the sensing optical fiber and in the reception and processing of its response, which is influenced by vibrations occurring in the proximity of the sensing fiber, [7], [8]. Procedures such as detection, localization, and even classification of vibration-type events alongside the sensing fiber path are based on such parameters as the latency, frequency spectrum, modulus, and response phase.

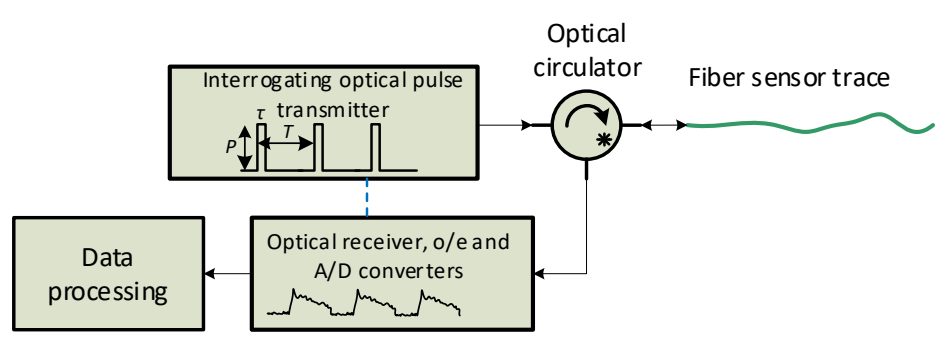

Figure 1: The basic principle of distributed fiber optic sensor.

The basic functional scheme of a distributed optic fiber vibration sensor based is shown in Figure 2. The sensor consists of a laser source, (whose light emission is controlled by a pulse generator), a laser protection isolator, an optical coupler, a photodetector, an A/D converter, and a computer unit for processing the acquired data, [7].

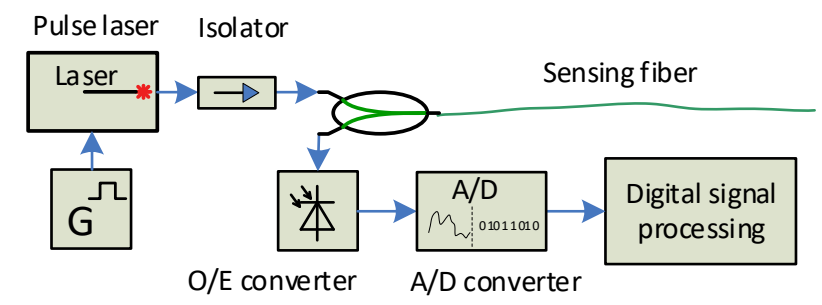

Figure 2: Basic functional diagram of a fiber optics distributed sensor.

The disadvantage of this basic sensor structure is the low stability of the pulsed laser, which results in low sensor performance, i.e., mainly the maximum sensing fiber length and the sensitivity. This disadvantage can be solved by the separating the laser beam generation, the optical pulse generation and the optical power amplification. In such a case, a continuous-wave, low-power, and highly stable laser can be used, pulses are generated by an optical modulator and the required power is got from an optical amplifier. A further improvement is achieved by replacing the optical coupler with an optical circulator with lower coupling losses and higher isolation. The most commonly used version is shown in Figure 3. It consists of several costly components, where the most expensive are ultra-narrow band laser, power optical amplifiers, acousto-optic modulator, optical preamplifier, balanced photodetector, and some others. 


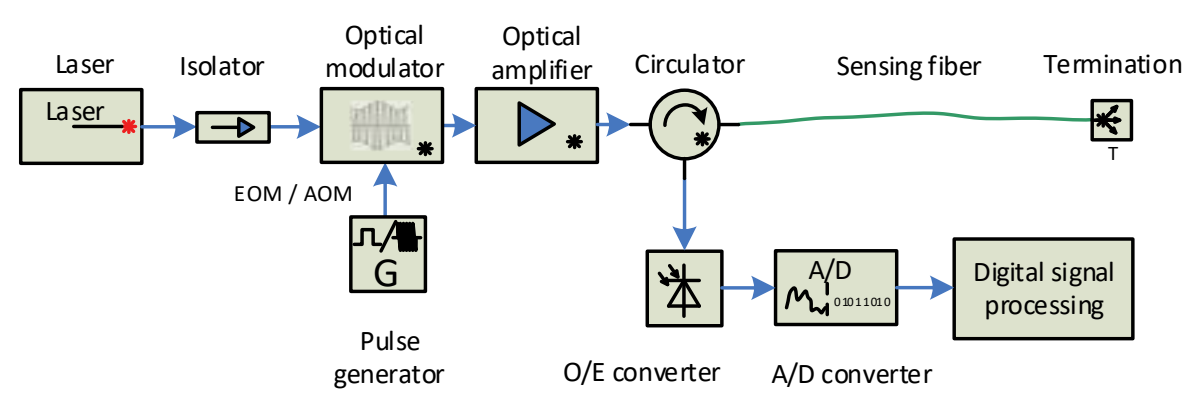

Figure 3: Functional diagram of a distributed fiber optic sensor.

Therefore, modifications of the basic connection leading to more efficient use of expensive components are beneficial. Better efficiency may be achieved by expanding the applicability of equipment or the number of functions while ensuring the required values of qualitative indicators provided by distinguished parts.

\section{Range extension and sensitivity improvements}

Optical pulses entering an optic fiber carry energy whose amount is set by the transmission power and the width of the pulses. Both the optic pulse power level and the fiber response level exponentially decrease during their propagation in the fiber. As a result, the received signal (backscattered fiber response) is attenuated with a coefficient equal to twice the optic signal attenuation coefficient in the fiber. A photodetector designed for appropriate wavelength band and frequency bandwidth is based on a photodiode and an electric transimpedance amplifier. These components determine the converter sensitivity, and it is limited by the overall level of the noise sources present in both optical and electrical parts of the receiver.

There are several basic options available to extend the sensor range and to increase its sensitivity:

a) Increasing the power of interrogating optic pulses - this technique can be applied up to a certain level only. Further increase of the optical pulse power may cause nonlinear effects (Brillouin effect, self-phase modulation, and others.).

b) Increasing the width of optical pulses - this results in an inversely proportional reduction of the sensor spatial resolution.

c) Amplification of the optical signal - it is possible to amplify the power of either the interrogating signal or the fiber response signal or both:

a. the signal amplification on sensing fiber - applying optical amplifiers on the sensing fiber must allow two-way propagation of the optical signal

i. in-line optical amplifiers - bidirectional optical amplifiers are used at certain distances. In addition to bidirectionality demand, the amplifierproduced ASE (Amplified Spontaneous Emission) noise is also a drawback that is difficult to mitigate.

ii. Distributed optical amplification - utilization of the Raman (single-stage or multi-stage one) or Brillouin amplifiers.

b. Amplification at the receiver path - amplifying the sensing fiber response in the optical part of the sensor receiver - unidirectional discrete or distributed amplifiers can be used.

d) Heterodyne receiver - employing a strong signal of the so-called local oscillator, merging with a weak response from the sensing fiber and subsequent conversion to 
electrical form, preferably by utilization of a balanced photodetector is obtained a product, the so-called resonant signal, whose level is given by the product of signal levels of both of the optical signals entering the converter. Due to the much stronger signal of the local oscillator, the resulting signal has a significantly higher level than the signal obtained by direct detection shown in Figure 3 . The beat signal product is further processed and finally converted to baseband. The structure of this improvement of signal detection is shown in Figure 4. This principle is very often used to increase the sensor sensitivity significantly, [12].

e) Separate processing of mutually perpendicular polarization planes of optical signals of the local oscillator and the fiber response - while using a heterodyne receiver, the gain of the heterodyne detection and following o/e conversion depends on the mutual polarization of the optical signals of the local oscillator and the response of the sensing fiber. If the mutual polarization of the two components of the optical signals is close to orthogonal, the effect of response amplification is suppressed. This leads to a loss of sensitivity of the sensor at specific points alongside the fiber. Therefore, due to the randomness of the polarization state of the sensing fiber response, it is appropriate to divide the fiber response into two mutually orthogonal polarization states, to split the local oscillator signal by the same way, and then to combine components with the same polarization state and to convert them into two electrical signals, which can then be combined to reduce the risk of sensitivity loss, and thus fail to capture the occurrence of events at specific points alongside the sensing fiber trace, [9].

The above modifications can also be combined in one device, as shown in Figure 5.

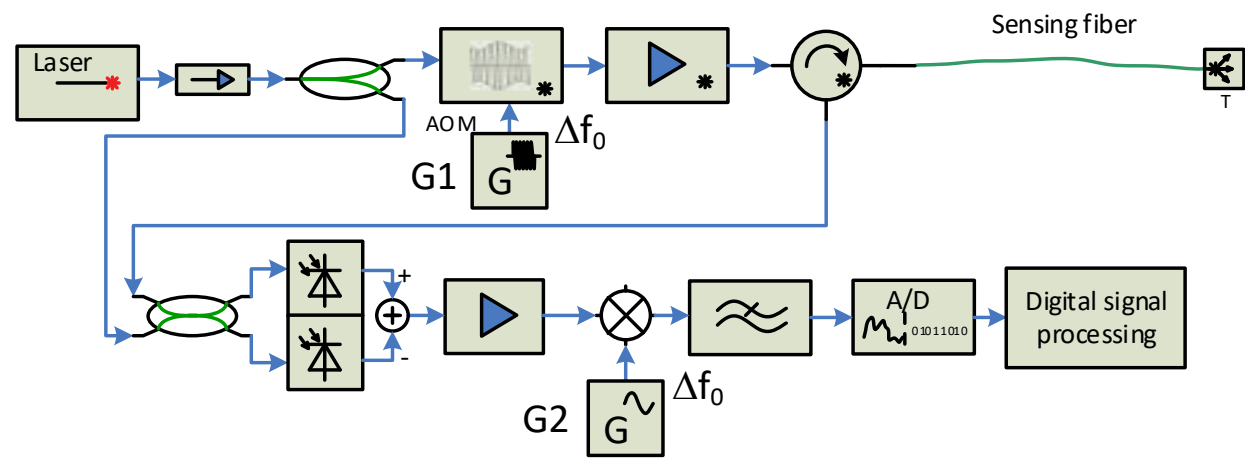

Figure 4: Schematic of a sensor with heterodyne receiver. 


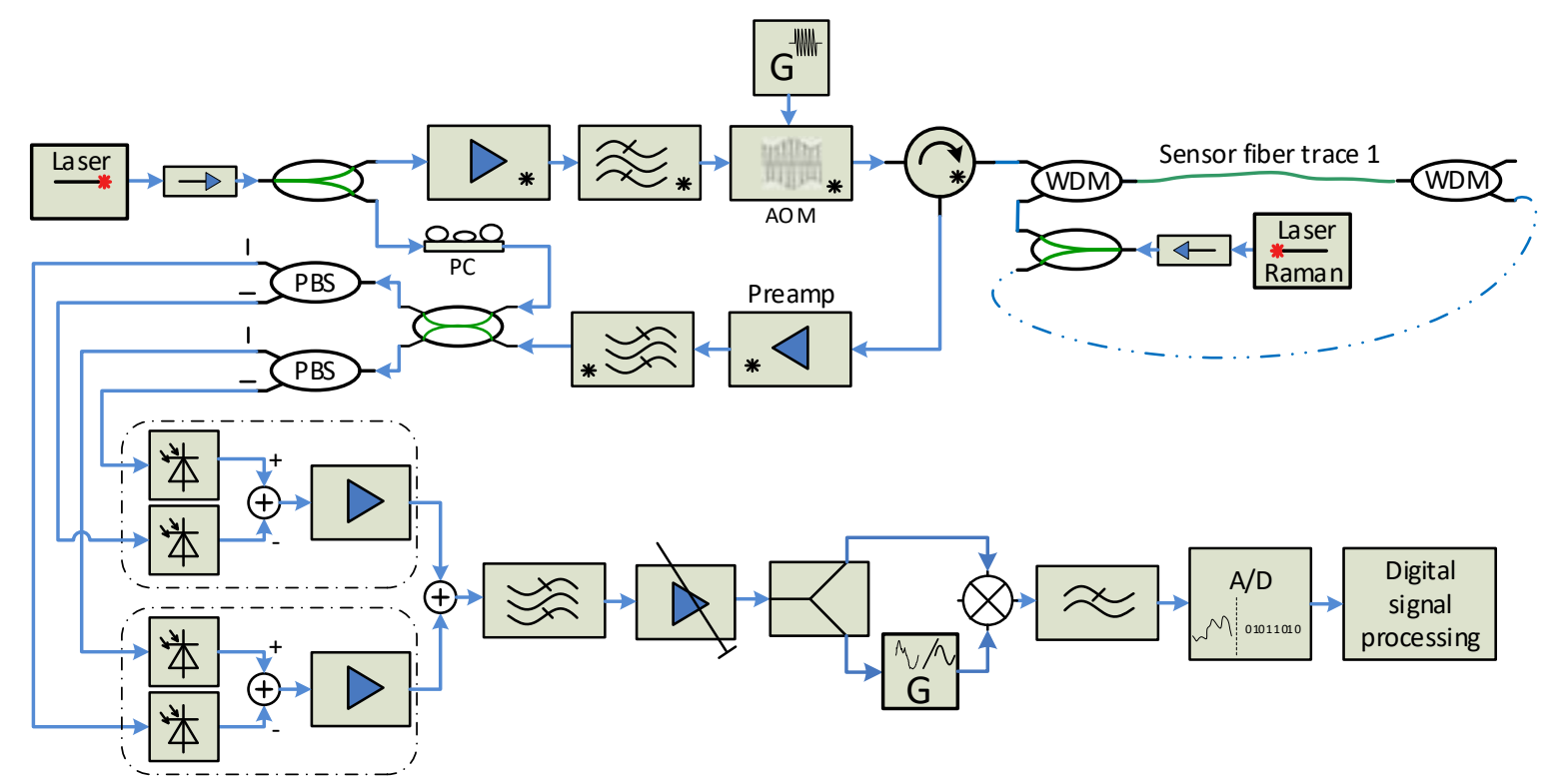

Figure 5: Sensor equipped with a heterodyne receiver, distributed optical amplifier (Raman amplifier), and separate processing of mutually orthogonal polarization planes.

The sensor on Figure 5 is a single-fiber sensor with a heterodyne receiver, Raman amplification (optionally with both forward and reverse pumping), with optical amplification in the receiving path (preamp and filtering), and polarization differentiation. The carrier for converting the beat signal to the baseband is recovered from the received signal.

In the following, the basic scheme for further modifications will be the system based on a heterodyne receiver as presented in Figure 4.

\section{Multi-fiber sensor unit}

A multi-fiber sensor means a sensor system operating on more than one sensing fiber using one measurement unit - a probe, (see Figure 6). If each sensing fiber represents a different trace, then events can be sensed along multiple paths to cover either a larger area or to provide multiple measurements in case of higher reliability requirements.

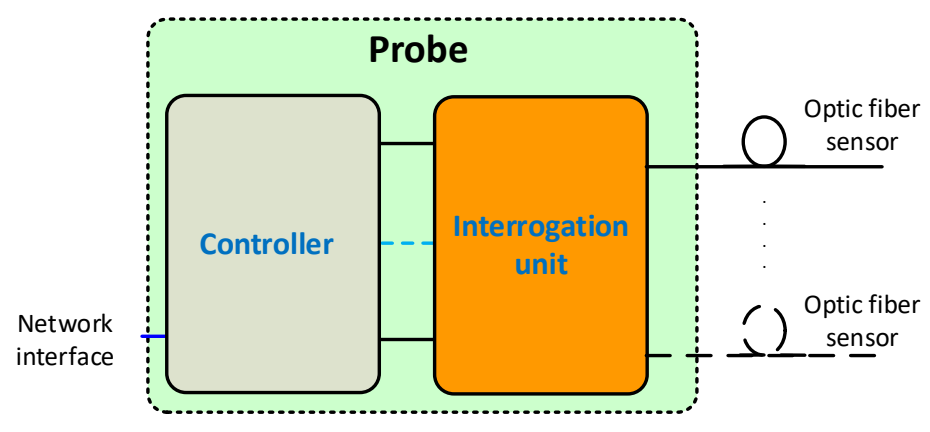

Figure 6: Multi-fiber sensor system principle.

Event monitoring on multiple sensing fiber paths can be:

a) Simultaneous - events on all $N$ sensing fibers are monitored simultaneously and independently of each other. This approach requires $N$ parallel chains of fiber response 
processing, including amplification, filtration, detection, localization, and classification. This results in the increase of the overall cost, the higher complexity of the system, and the demand for higher computing power. Despite of this the cost is lower than the adequate number of separate measurement units

b) Sequential - using an external optical switch, the measuring unit switches between several sensing fibers, where it performs a certain number of measurements on each trace. Switching can be either periodic or driven by some other rules, e.g., external trigger.

\section{Schemes for the concurrent scanning of multiple sensing fibers}

The primary type of a simultaneous multi-fiber sensor system with the heterodyne receiver is a measuring unit equipped with one source of optical pulses. Optical pulses consist of a laser, an optical power amplifier, a modulator, and an optionally optical filter for amplifier noise suppression. An example of a double-stranded version is shown in Figure 7. The optical pulse power is divided by an optical power splitter into several branches, which the individual sensing fibers are connected to. Because all branches use the same frequency band simultaneously, the sensor unit must contain the same number of receiving chains as the number of sensing fibers the signal is transmitted into. The local oscillator signal must also be split into the same number of branches to ensure heterodyne reception. The period of optical pulses transmission is the same for all sensing fibers regardless of their respective lengths and it is determined by the longest one.

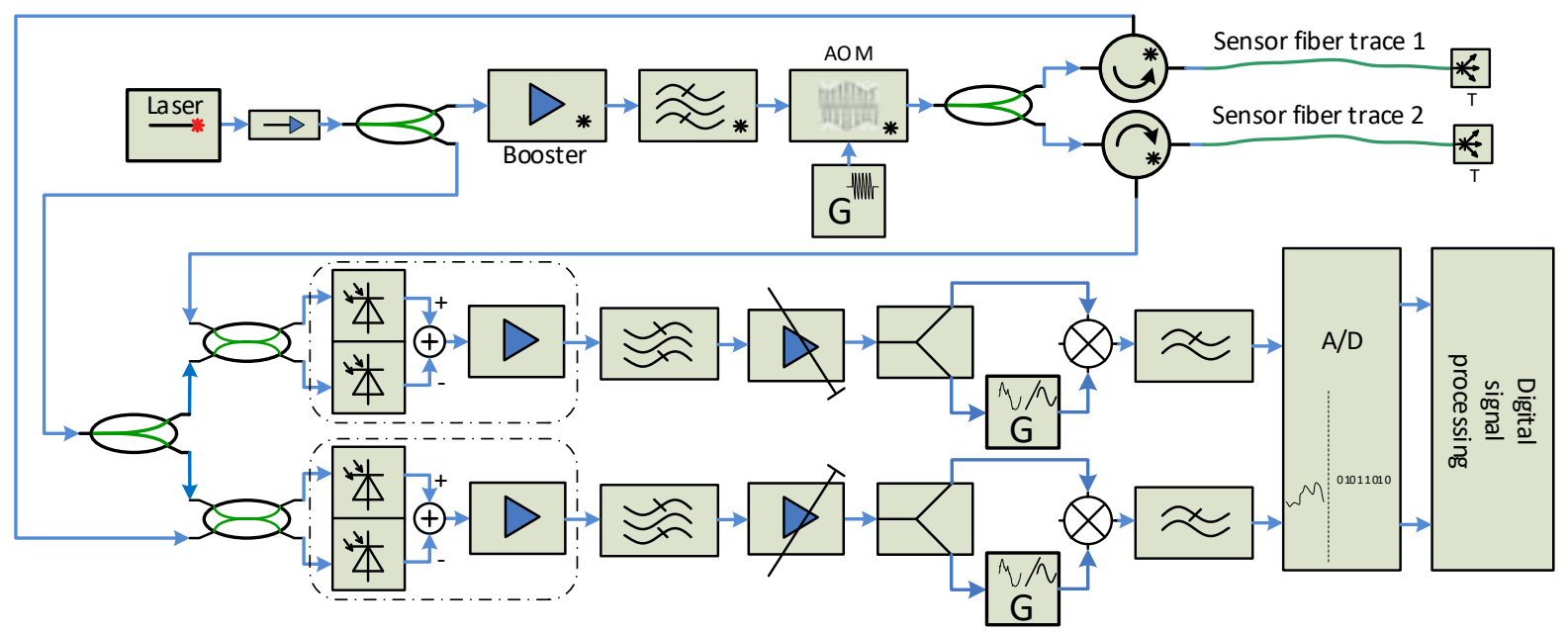

Figure 7: Two-fiber sensor unit (probe) with one modulator.

Suppose we use a dedicated acousto-optic modulator for each branch. In that case, we obtain independence in generating optical pulses for individual sensing fibers, unless it is limited by control pulse generators or data acquisition methods, i.e., by the D/A and/or A/D converters. In addition, if we choose modulators with non-overlapping carrier frequency bands, it is possible to combine the responses from several fibers into one complex signal and to realize a standard optical amplification and conversion to electrical form in one processing chain. This approach can save the number of amplifiers and balanced photodetectors (in the case of a twofiber unit from Figure 7, one component is saved, in the case of $N$ sensing fibers, $N-1$ photodetectors and amplifiers are saved). On the side of electrical signal processing, the complex signal must be split again, and the signals from distinguished sensing fibers have to 
be processed separately. The double-stranded form of such sensor system is presented in Figure 8 .

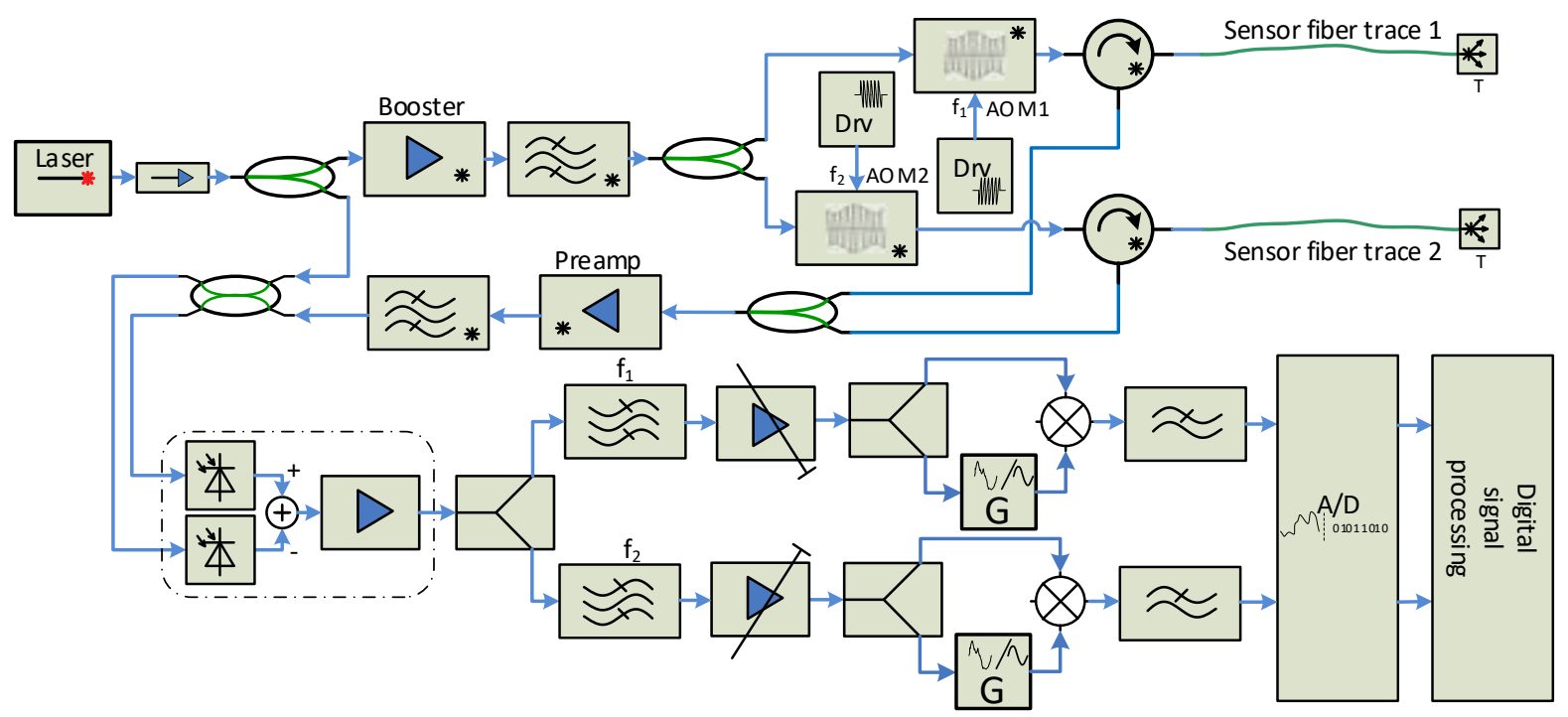

Figure 8: Dual-fiber form of the sensor unit with two modulators.

The drawback of the above solution is the need for several distinguished modulators and the signal attenuation due to the signal coupling and splitting. This requires use of additional amplifiers in the optical or electrical receiving parts or shorter sensing length. In the case of separate polarization processing the complexity of the sensor unit is further increased, as shown in Figure 9. 


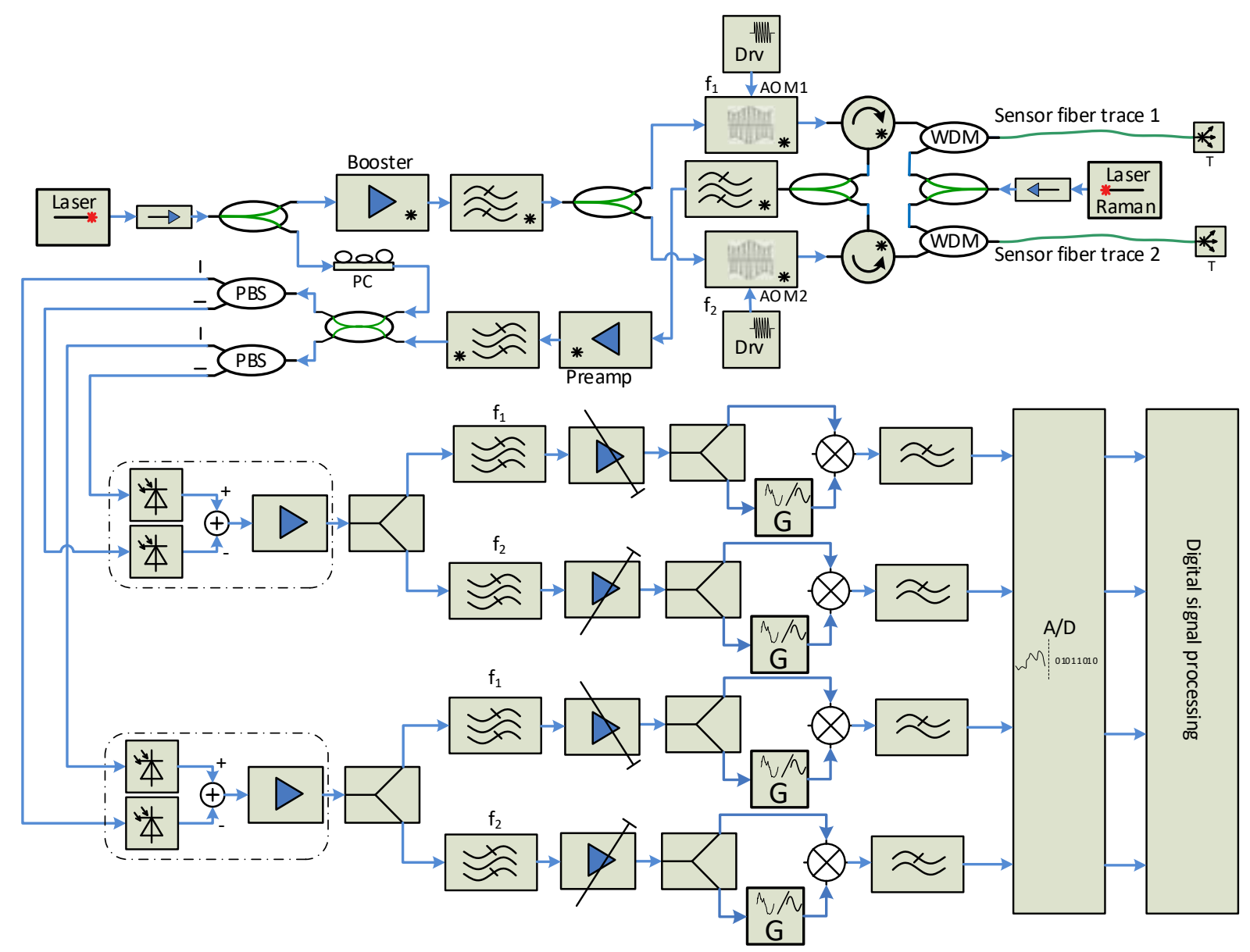

Figure 9: Dual-fiber form of the sensor unit with Raman amplification and polarization differentiation.

The forward path of the scheme is the same as in Figure 8 and extended by the Raman amplification. Raman pump power is split into two branches and combined with interrogating signal in the WDM coupler in each branch. The fiber responses are coupled and the pump scattered signal is filtered out before amplification and ASE noise filtering. Then after the signal is combined in a 50/50 optical coupler with the local oscillator signal, whose polarization is appropriately rotated to obtain approximately the same power after polarization splitting. The signals from both outputs of the 50/50 coupler is then passed through a polarization beam splitters (PBS), which splits the signal into two mutually orthogonal polarizations. The pair of PBS outputs with the same polarization enters the same balance photodetector. After conversion to electrical form, the signal of each photodetector is split into two branches, and the resulting four signals are further processed separately.

Both single-fiber and two-fiber sensor units were constructed and tested both in the laboratory, see Figure 10, ([31], [32]). Both sensor units in the picture are operating, waterfalls are shown on displays. Three fiber traces are $50 \mathrm{~km}$ long each and bare fiber spools are used (one is shown on the left).

The two-fiber sensor contains the Raman amplifier and it has 4-channel data acquisition card. It supports several configurations, e.g. to send two pulses with non-overlapping AOM frequency shifts to the fiber or to process mutually orthogonal polarizations from two fibers simultaneously, according to scheme depicted in Figure 9. Thus the two-fiber sensor unit can cover approximately $200 \mathrm{~km}$ range. 


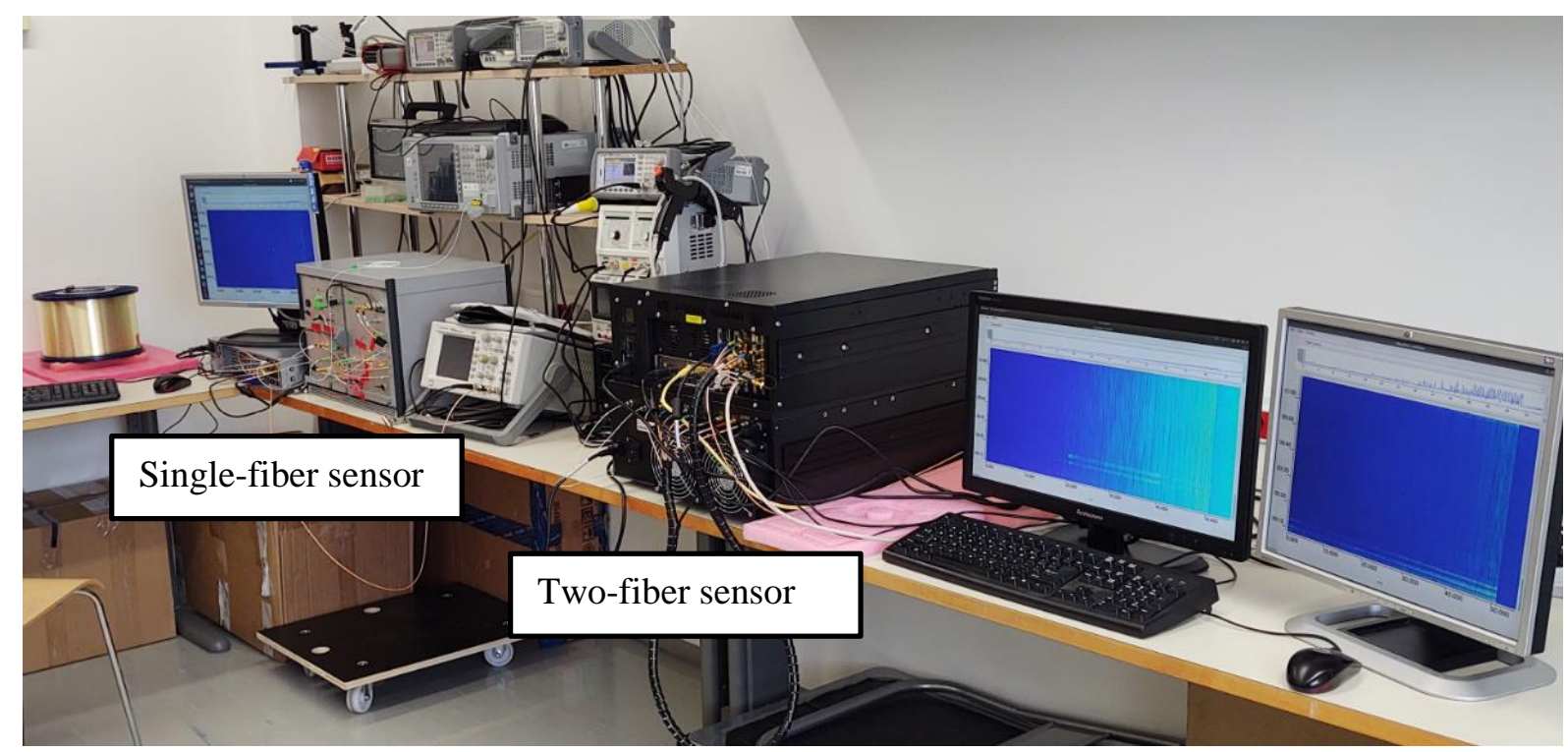

Figure 10: Two sensor units (single-fiber and two-fiber) in operation - testing in the laboratory

The sensor units were also connected to the real fiber traces buried approx. $50 \mathrm{~cm}$ and $1 \mathrm{~m}$ under ground surface. Several different events producing mechanical vibrations were generated. Two cases are depicted in Figure 11.

a)

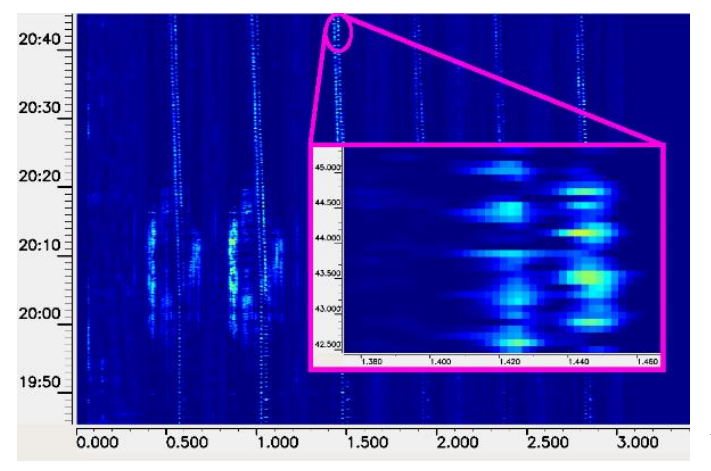

b)

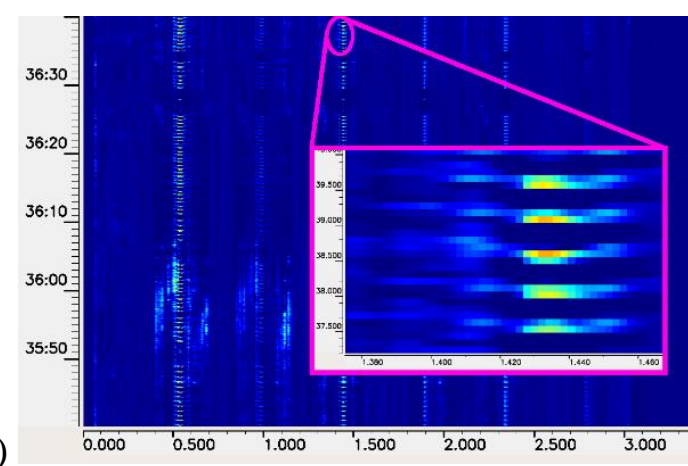

Figure 11: Sensor testing in a real setup: a) a walk of two persons spaced 20 meters, b) hammering the rod into the ground

\section{Sequential scanning of multiple sensing fibers}

In the sequential scanning of several sensing fiber traces, the sensor unit is switched among $N$ sensing fibers using an external 1: $N$ optical switch (see Figure 12). Sharing one probe with more sensing fiber traces provides more efficient usage of the expensive probe equipment.

The sensor unit is set to generate certain number of measurement pulses per unit time, which is limited by the longest sensing fiber or by the sensor itself. The frequency is thus the same for all sensing fibers. Switching among fibers can be either periodic or driven by any other rule. The measurement is blocked during switching phases. Important parameters, which determine the overall number of scans per switching period, are the switching period duration, the switching latency and the number of switches per the period. 


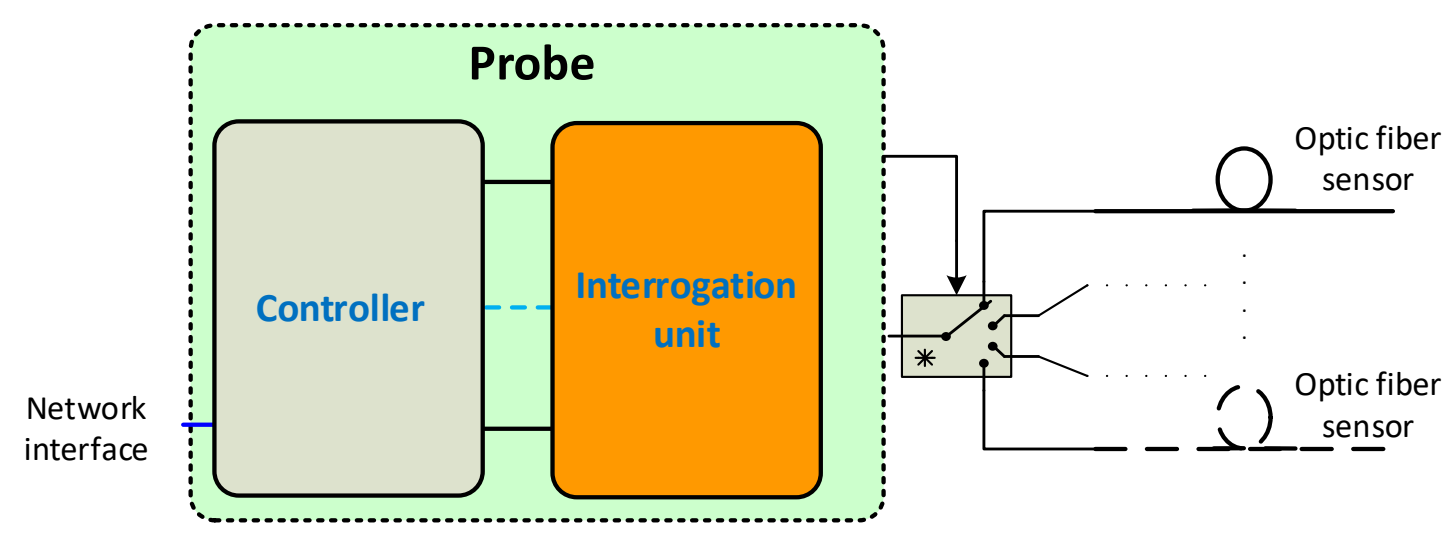

Figure 12: Probe sharing among several sensing traces

Therefore, a value expressing the number of required scans per unit time and per the sensing fiber must be determined and the distribution of scans within a time unit to obtain enough data from each line for further processing. The amount of data needed depends on whether the sensor units only need to detect and locate the vibration sources or whether it is also necessary to identify (classify) the sources.

There are two main types of fiber optic switches: MEMS (micro-electromechanical system) based and switches based on a magneto-optical principle. The typical switching time of MEMS-based switches is about $0.5-1 \mathrm{~ms}$. The switching time depends on the case whether the switching occurs between directly neighboring ports or not.. The typical switching time of magneto-optic switches is in order of units of $\mu$ s. The price of the fiber optic switch matters as well. The price of magneto-optical switch is typically 3-4 times higher than the price of a MEMS-based switch with the same number of ports. The optical switch can have more applications in distributed vibration sensors; namely, they can improve the flexibility of the sensor unit.

\section{Utilization of optical switches for sensor unit flexibility improvement}

When deploying multi-fiber sensor systems, not necessarily all sensing ports must be always used, i.e. connected to sensing fibers. Therefore, it is appropriate to design the sensor units concerning efficient use of outputs of signal generating subsystem. This means that no energy is wasted for unused ports. It allows more efficient optical energy usage to active ports, which have sensing fibers connected to. The modification will be demonstrated on two-fiber systems.

Figure 13 and Figure 14 show two schemes of a two-fiber sensor units with the heterodyne receiver described in the chapter "Concurrent scanning of multiple sensing fibers" (see Figure 7 and Figure 8). These setups are equipped with one and two optical modulators, respectively, and with Raman signal amplification circuit. The aim is to modify these setups to efficiently use optical signals for both two fiber and one fiber deployments. 


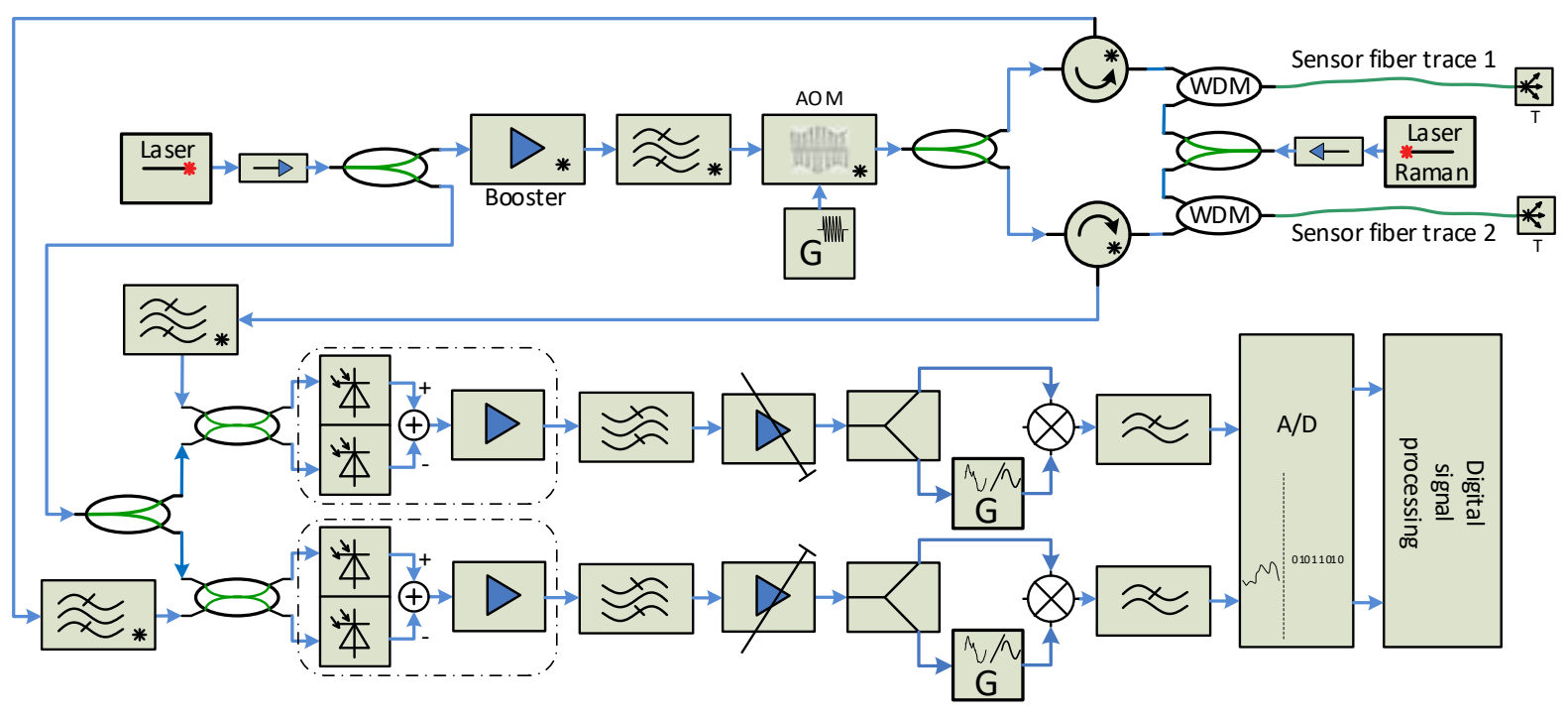

Figure 13: Two-fiber sensor unit with one modulator and Raman amplifier.

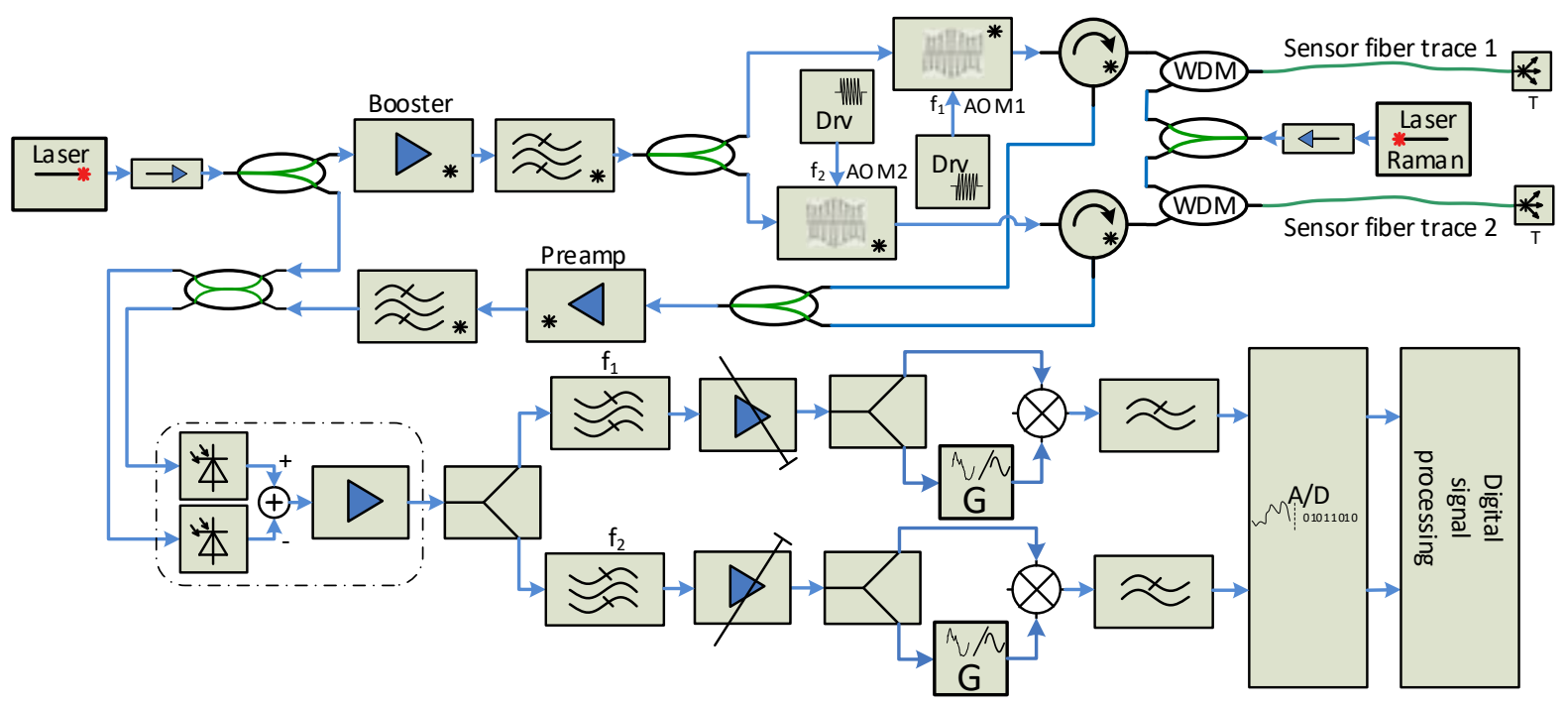

Figure 14: Two-fiber sensor unit with two modulators and Raman amplifier.

In the setup with one optical modulator (see Figure 13), an optical coupler 2:2 will replace the originally proposed 1:2 optic splitter, and a 2-port switch is connected to the coupler second output (see Figure 15). In the case of the deployment with one sensing fiber only, this setup allows sending the signal from the unused output back to second coupler input and thus to strengthen signal at the active output.

a)
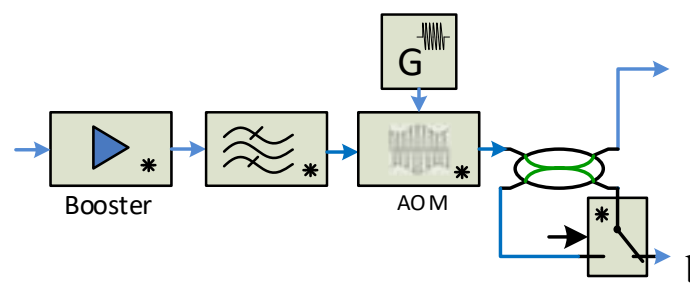

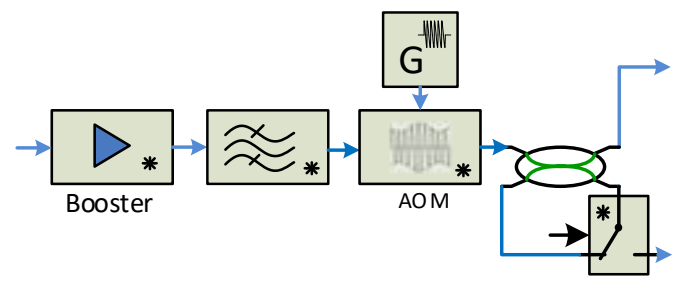

Figure 15: A scheme for efficient utilization of optical pulse power in a dual-fiber sensor system: a) two active sensing fibers; $b$ ) single active sensing fiber. 
When the sensing system contains two modulators with non-overlapping frequencies (see Figure 14) more options are available and therefore more flexible modification of the original setup can be designed (see Figure 16).

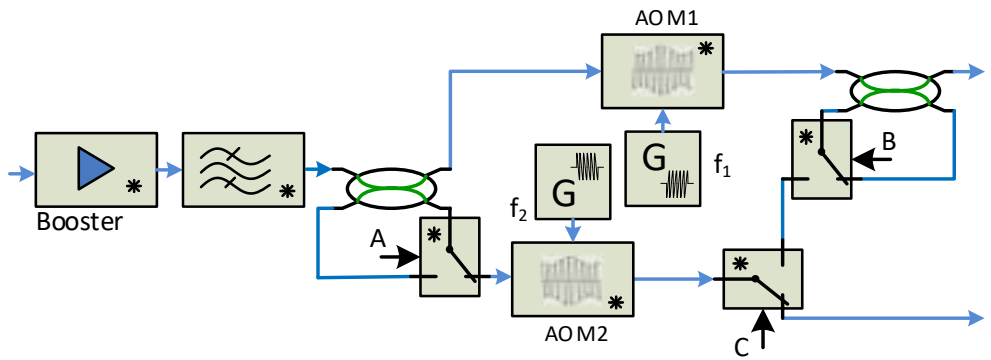

Figure 16: Modification of the transmission chain of a two-fiber sensor system with two modulators offering better flexibility.

Splitting of the optical amplifier (booster) output is solved by the same way as described above in the case of one modulator system. By utilizing two additional two-port switches behind the AOM modulators other options are possible. When both sensing fibers are connected the position of both switches are as shown in Figure 16. If only one sensing fiber is used, either only AOM1 branch is active and switch A changes its position or both AOM1 and AOM2 branches are active (switch $\mathrm{A}$ is in its original position) and the AOM2 output is switched through switches $\mathrm{B}$ and $\mathrm{C}$ to the sensing trace 1 . This causes that two pulses at different frequencies are sent to the same sensing fiber. Both pulses may have different widths and different instants of generation too. As the result it can improve the sensor's sensitivity fading issue.

To prolong the range of sensing the Raman amplification is frequently used. One setup is shown in Figure 13 and in Figure 14.

In both original schemes, the Raman pump output power is distributed evenly to both ports via WDM couplers. If the Raman pump is in operation and only one sensing fiber is applied, a half of the pump output power normally would be lost. In addition, it would be necessary to terminate the unused port with an optical terminator for absorption of relatively high pump power (hundreds of $\mathrm{mW}$ ). The unterminated port can be very risky for human or animal health if they focus their eyes at the unterminated port, which the strong pump signal is leaking from. The solution enabling the efficient use of the pump power and other deployment options are presented in Figure 17 and in Figure 18.

a)

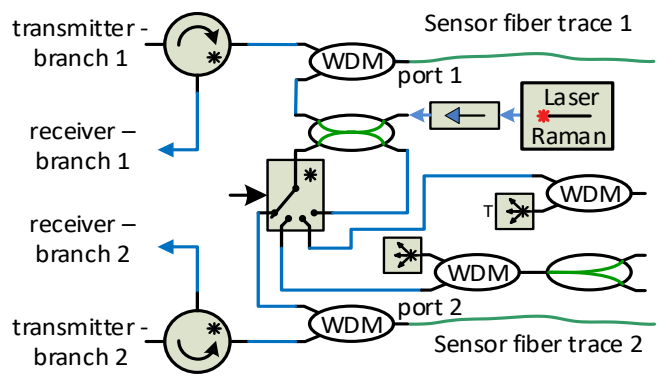

b)

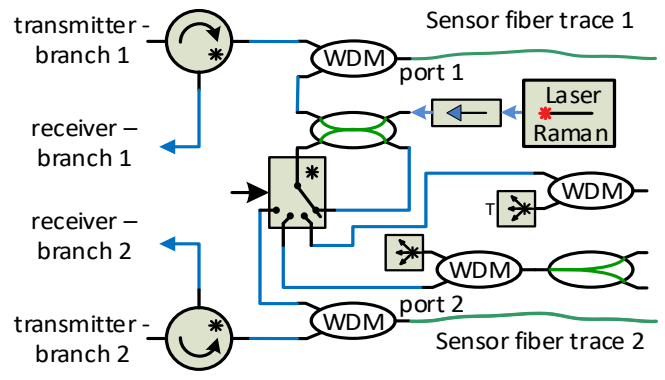

Figure 17: Modification of the Raman pump connection for the sensor flexibility improvement: a) balanced Raman pump distribution, b) Raman pump power concentration for the fiber trace 1 
To enable flexible and efficient usage of two-port sensor unit a 4-port switch was implemented into both schemes (see Figure 17 a). If both sensing traces are used the switch position is as shown in Figure 17 a) and a half of pump power is sent to the fiber 1 and the second half to the fiber 2 .

If only one sensing fiber trace is required, the sensing fiber will be connected to the sensor fiber trace 1 (see Figure 17) and thus the port 1 is active. The 4-port optic switch changes its position to one shown in Figure $17 \mathrm{~b}$ ), and the pump power originally directed to the port 2 (which is now unused) is redirected (with minor loss) back to the port 1 for forward Raman amplification.

Alternatively, if both ends of the sensing fiber of trace 1 are terminated at the place where the sensing unit (the probe) is located, the second output of the coupler with Raman pump power can be switched to the second end of the trace 1 for backward amplification (see Figure $18 \mathrm{a}$ ). The fiber trace 2 is without Raman amplification either active or optionally unused. Figure 18 b) shows the other option with the same switch position where both ports are active and the fiber trace 1 is forward pumped and the fiber trace 2 is backward pumped.

If both ports are active and both of them begin and end at the place of the probe, it is possible to use the switch to split and forward the second half of the pump power in a counterpropagating direction of both sensing fibers (see Figure $18 \mathrm{c}$ ). Thus the 4-port switch implementation extends one original scheme to five optional schemes.

a)

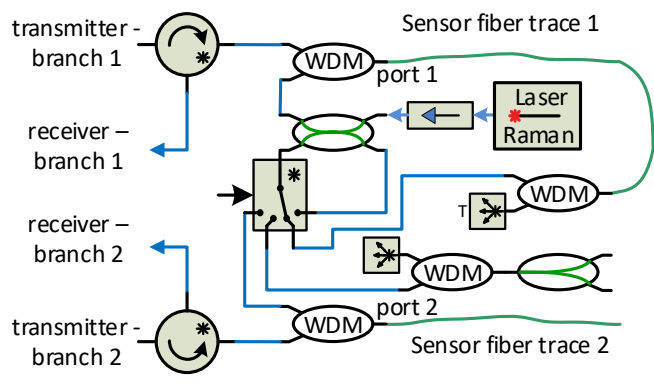

c)

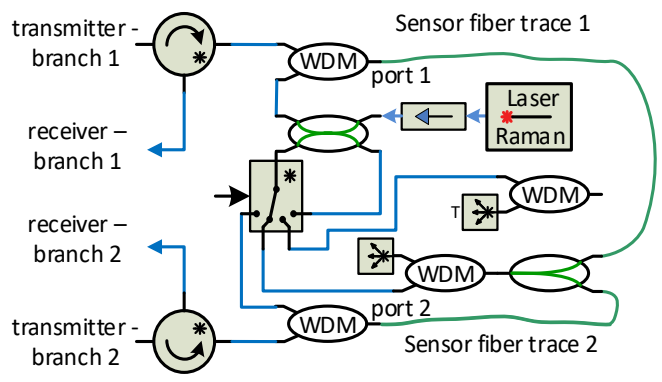

b)

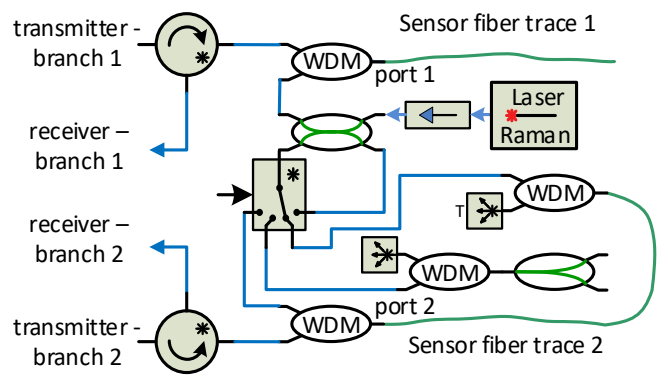

Figure 18: Three additional options of the sensor unit application with 4-port switch implementation

The overall solutions of flexibility improvement of the sensor unit transmitting parts is shown in Figure 19 and Figure 20. 


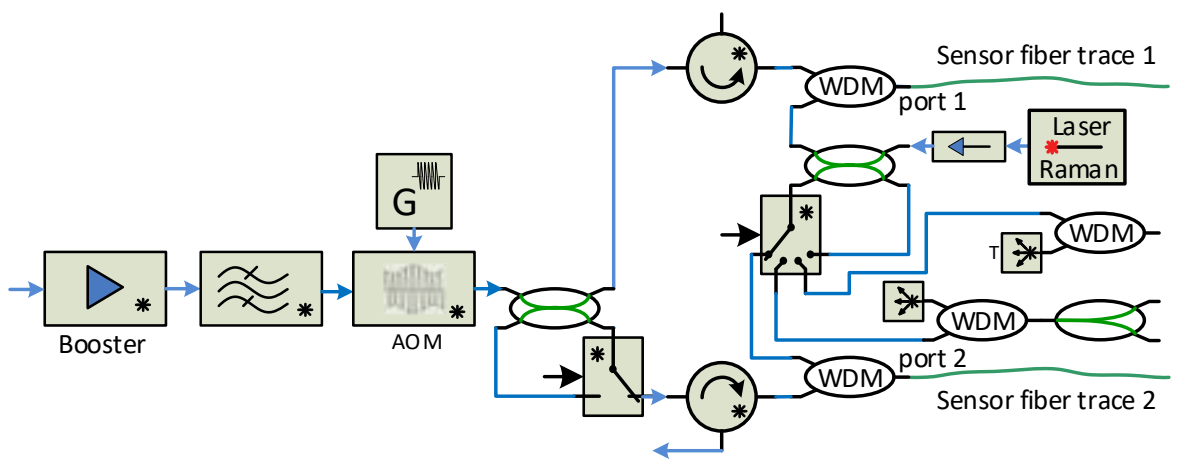

Figure 19: Utilization of optical switches for a two-fiber sensor with one modulator and a Raman amplifier.

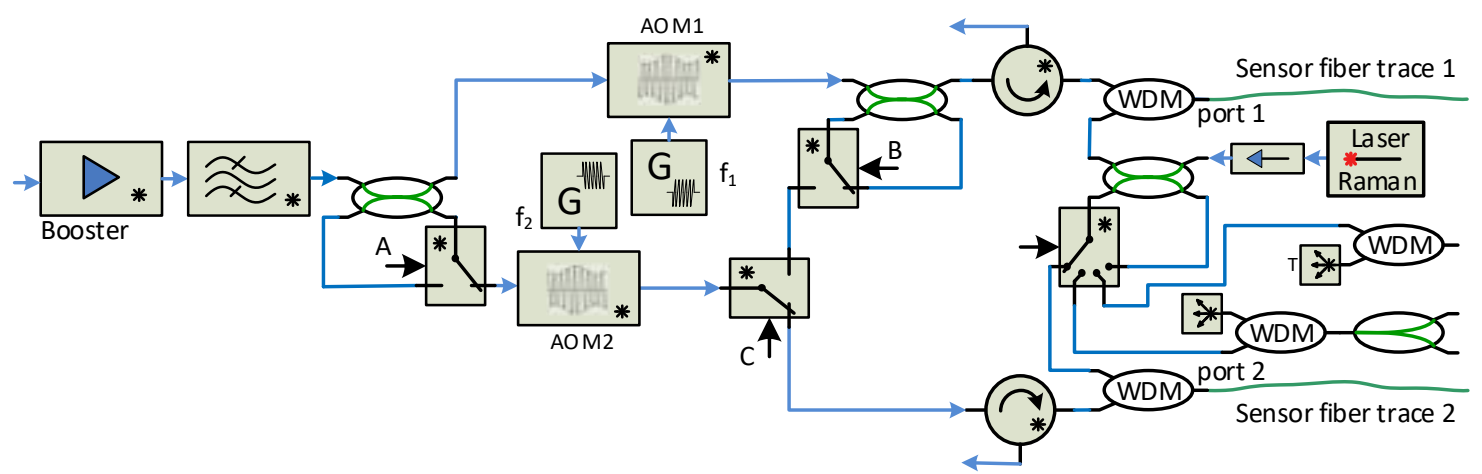

Figure 20: Utilization of optical switches for a two-fiber sensor with two modulators and a Raman amplifier.

\section{Conclusion}

There are a wide variety of modifications of the distributed fiber optic sensor basic scheme that try to improve its performance or to obtain more flexible design. Some of them have been presented in this paper. Their main benefits are streamlined utilization of the expensive heart of the sensor - laser, optical amplifiers, modulators, and broader sensor system application opportunity.

Like many similar attempts to expand the device's functionality or to further improve its parameters, these modifications also have their pitfalls. Namely, the complexity increase of the setup, sometimes increasing the cost due to the need for additional components, and thus increasing the likelihood of complications in their daily operations.

\section{Data Availability}

The data used to support the findings of this study are included within the article. Further information is available from the corresponding author upon request.

\section{Conflicts of Interest}

The authors declare that there is no conflict of interest regarding the publication of this paper. 


\section{Funding Statement}

This research was funded by Ministry of Interior of the Czech Republic under grant no. VI20172020078.

\section{References}

[1] J. S. Bhavin and D. V. Plant, "Scaling Technologies for Terabit Fiber Optic Transmission". Proc. of SPIE, Optoelectronic Integrated Circuits XIII, Vol. 7942, pp. 1-12, doi:10.1117/12.880247, 2011.

[2] E. Udd. W.; B. Spillman Jr. "Fiber Optic Sensors: An Introduction for Engineers and Scientists," (Second edition). John Wiley \& Sons, ISBN 978-0-470-1264-4, New Jersey, USA, 2011

[3] Q. Chai, Y. Luo, J. Ren, et al. "Review on fiber-optic sensing in health monitoring of power grids," Optical Engineering, 58(7) 072007, https://doi.org/10.1117/1.OE.58.7.072007, 2019

[4] M. R. Fernández-Ruiz, M. A. Soto, E. F. Williams, et al. "Distributed acoustic sensing for seismic activity monitoring”. APL Photonics [online]. 2020, 5(3). ISSN 2378-0967. Available at: doi:10.1063/1.5139602

[5] Ch. Wiesmyr, M. Litzenberg, et.al. "Real-Time Train Tracking from Distributed Acoustic Sensing Data”. Applied Sciences [online]. 2020, 10(2). ISSN 2076-3417. Available at: doi:10.3390/app10020448

[6] A. Barrias, J. Casas and S. Villalba. "A Review of Distributed Optical Fiber Sensors for Civil Engineering Applications”. Sensors [online], 16(5) [cit. 2021-5-16]. ISSN 1424-8220. Available at: doi:10.3390/s16050748, 2016.

[7] H. F. Taylor and C. E. Lee, “Apparatus and method for fiber optic intrusion sensing," U.S. Patent 5 194847, Mar. 16, 1993

[8] J. C. Juarez, E. W. Maier, K. N. Choi, and H. F. Taylor, "Distributed fiber-optic intrusion sensor system," J. Lightwave Technology 23(6), 2081-2087, 2005.

[9] J.C. Juarez, and H.F. Taylor, "Polarization discrimination in a phase-sensitive optical time-domain reflectometer intrusion-sensor system". Optics Letters, 30(24): pp. 3284-3286. 2005

[10] S. M. Maughan "Distributed Fiber Sensing Using Microwave Heterodyne Detection of Spontaneous Brillouin Backscatter" (Doctoral thesis). University of Southampton, 2001, UK.

[11] C. K. Madsen, T. Snider, R. Atkins, J. Simcik, "Real-time processing of a phase-sensitive distributed fiber optic perimeter sensor," in Proceedings of the SPIE, Sensors, and Command, Control, Communications, and Intelligence (C3I) Technologies for Homeland Security and Homeland Defense VII, vol. 6943, no. 6943-36, 2008.

[12] Y. Lu, T. Zhu, L. Chen, and X. Bao, "Distributed vibration sensor based on coherent detection of phase-OTDR,” J. Lightwave Technol. 27, 3243-3249, 2010.

[13] B. Szafraniec, T. S. Marshall and B. Nebendahl "Performance Monitoring and Measurement Techniques for Coherent Optical Systems.” Journal of Lightwave Technology [online]. 31(4), 648-663. ISSN 0733-8724. Available at: doi:10.1109/JLT.2012.2212234, 2013

[14] F. Gaosheng, T. Xu, F. Shengwen and F. Li "Phase-Sensitive Optical Time Domain Reflectometer Based on Phase-Generated Carrier Algorithm.” Journal of Lightwave Technology. Vol. 33, pp. 28112816. 2015

[15] Z. Pan, K. Liang, J. Zhou, et al. "Interference-fading-free phase-demodulated OTDR system" Proc. of OFS2012 22nd International Conference on Optical Fiber Sensors, 842129 (17 October 2012); https://doi.org/10.1117/12.975656

[16] H. L. Byeong,H. K. Young, S. P. Kwan, et al. "Interferometric Fiber Optic Sensors”. Sensors, ISSN 1424-8220, pp. 2467-2486, 2012

[17] L. Thénevaz, "Brillouin distributed time-domain sensing in optical fibers: state of the art and perspectives" [online]. Higher Education Press and Springer-Verlag Berlin Heidelberg 2010 URL: http://infoscience.epfl.ch/record/143879

[18] H. F. Martins, S. Martín-López, P. Corredera, M. L. Filograno, O. Frazão and M. Gonzalez-Herráez, "Phase-sensitive Optical Time Domain Reflectometer Assisted by First-order Raman Amplification for Distributed Vibration Sensing Over $>100$ km,". In Journal of Lightwave Technology, vol. 32, no. 8, pp. 1510-1518, doi: 10.1109/JLT.2014.2308354, 2014.

[19] F. Peng, H. Wu, X. H. Jia, Y. J. Rao, Z. N. Wang, Z. P. Peng „Ultra-long high-sensitivity $\Phi-O T D R$ for high spatial resolution intrusion detection of pipelines“. Opt. Express. 2014 Jun 2;22(11):13804-10. doi: 10.1364/OE.22.013804. PMID: 24921572, 2014. 
[20] Z. Qin, H. Chen and J. Chang, "Detection Performance Improvement of Distributed Vibration Sensor Based on Curvelet Denoising Method”. Sensors [online]., 17(6). ISSN 1424-8220. Available at: doi:10.3390/s17061380, 2017.

[21] R. Meiqi, "Distributed Optical Fiber Vibration Sensor Based on Phase-Sensitive Optical Time Domain Reflectometry". MS.C. thesis at University of Ottawa, 2016.

[22] J. Xiong, Z. Wang, Y. Wu and Y. Rao, "Single-Shot COTDR Using Sub-Chirped-Pulse Extraction Algorithm for Distributed Strain Sensing," in Journal of Lightwave Technology, vol. 38, no. 7, pp. 2028-2036, doi: 10.1109/JLT.2020.2968632, 2020.

[23] R. Brandon, M. J. Murray, A. Davis a Clay Kirkendall. "Quantitative amplitude measuring $\varphi$-OTDR using multiple uncorrelated Rayleigh backscattering realizations”. Optics Express [online], 27(24). ISSN 1094-4087. Available at: doi:10.1364/OE.27.034952, 2019.

[24] Wang, Z., L. Zhang, S. Wang, et al. "Coherent $\Phi$-OTDR based on I/Q demodulation and homodyne detection”. Optics Express [online], 24(2). ISSN 1094-4087. Available at: doi:10.1364/OE.24.000853, 2016.

[25] S. Liehr Y.S. Muanenda, S. Münzenberger, K. Krebber "Relative change measurement of physical quantities using dual-wavelength coherent OTDR". Optics Express, 25(2):720-729. doi: 10.1364/OE.25.000720. PMID: 28157961, 2017.

[26] H. He, L.-Y. Shao, Z. Li, et al. "Self-Mixing Demodulation for Coherent Phase-Sensitive OTDR System". Sensors, 16, 681. https://doi.org/10.3390/s16050681, 2016.

[27] X. Liu, B. Jin, Q. Bai, Y. Wang, D. Wang and Y. Wang "Distributed Fiber-Optic Sensors for Vibration Detection". Sensors [online], 16(8). ISSN 1424-8220. Available at: doi:10.3390/s16081164, 2016.

[28] B. Xiaoyi, D.-P. Zhou, Ch. Baker and L. Chen. "Recent Development in the Distributed Fiber Optic Acoustic and Ultrasonic Detection". Journal of Lightwave Technology [online], 35(16), 3256-3267. ISSN 0733-8724. Available at: doi:10.1109/JLT.2016.2612060, 2017.

[29] R. Zinsou, , L. Xin , Y. Wang, et al. "Recent Progress in the Performance En-hancement of PhaseSensitive OTDR Vibration Sensing Systems,". Sensors, 19(7). ISSN 1424-8220. Available at: doi:10.3390/s19071709, 2019.

[30] Z. Wang, B. Lu, Q. Ye, and H. Cai, "Recent Progress in Distributed Fiber Acoustic Sensing with $\Phi$ OTDR”. Sensors [online]., ISSN 1424-8220. doi:10.3390/s20226594, 2020.

[31] V. Novotny, P. Sysel, "Long-Range Optical Fibre Based Distributed Vibration Sensing”. In Proceedings of the KTTO 2016 conference proceedings, pp. 38-43. ISBN: 978-80-248-3959- 2, 2016.

[32] V. Novotny, P. Sysel, J. Prinosil, J. Mekyska, K. Slavicek, and I. Lattenberg, I. "Critical Infrastructure Monitoring System”. In: 2021 IEEE 17th International Colloquium on Signal Processing \& Its Applications (CSPA) [online]. IEEE, 2021-3-5, s. 165-170. ISBN 978-1-6654-1484-5, doi: 10.1109/CSPA52141.2021.9377303, 2021. 